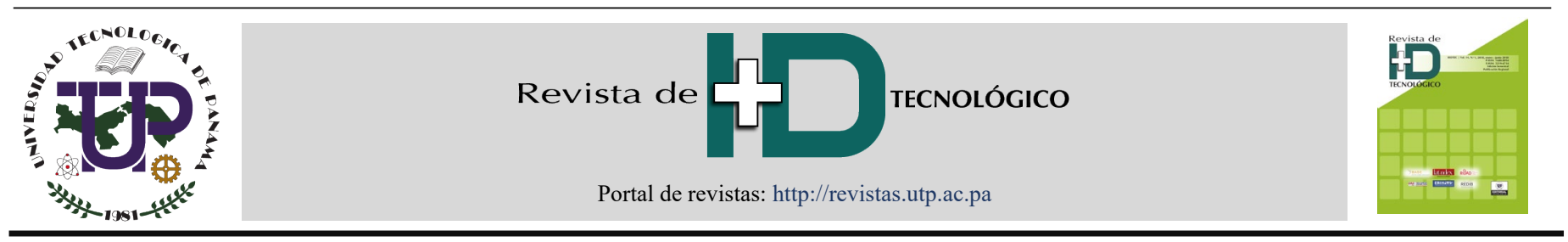

\title{
Efecto de las islas de calor urbano en las principales vías de la Ciudad de Panamá
}

\section{Urban heat island effect on the main roads of Panama City}

\author{
Martín Candanedo ${ }^{1}$, Danna Villarreal ${ }^{{ }^{*}}$ \\ ${ }^{1}$ Facultad de Ingeniería Civil, Universidad Tecnológica de Panamá, Panamá \\ *Autor de correspondencia: danna.villarreal@utp.ac.pa
}

RESUMEN- Las islas de calor urbano se definen como la diferencia de temperatura en un área urbana en comparación de sus alrededores. Estas pueden ser causadas por un alto consumo eléctrico, uso excesivo de automóviles, diseño y planificación de infraestructuras y los materiales utilizados, y la disminución del área verde. Debido a su presencia en distintas ciudades del mundo y analizadas con estudios pertinentes, se decide realizar un estudio en las principales vías de la Ciudad de Panamá. Se plantea investigar la existencia de islas de calor y sus posibles causas, debido a su desarrollo urbano no planificado. Se delimitan cuatro zonas de estudio para recolectar datos de temperatura con un termo higrómetro en un horario de 8:00 am, 2:00 pm y 8:00 pm en temporada de transición, lluviosa y seca. Consiguiente, se elaboraron mapas de isotermas utilizando el método de EBK. Como resultado se obtuvieron diferencias de temperaturas máximas y mínimas de $9^{\circ} \mathrm{C}$ para el período matutino, $8.1^{\circ} \mathrm{C}$ para la tarde y $2.4^{\circ} \mathrm{C}$ para la noche para la temporada de transición; los valores de $10.5^{\circ} \mathrm{C}$ para la mañana, $8.1^{\circ} \mathrm{C}$ para la tarde y $3.3^{\circ} \mathrm{C}$ para la noche en temporada lluviosa; y por último la temporada seca con $7.8^{\circ} \mathrm{C}$ para el horario matutino, $9^{\circ} \mathrm{C}$ para el vespertino y $2.6^{\circ} \mathrm{C}$ para el nocturno. Se comprueban las islas de calor y su relación con edificios altos, uso de automóviles, el uso de asfalto como material para las calles y factores ambientales como la lluvia, nubes y vientos promueven la generación de ellas.

Palabras clave-Islas de calor urbana, temperatura, Ciudad de Panamá, Empirical Bayesian Kriging.

ABSTRACT - Urban heat island effect is defined as the temperature difference in an urban area compared to its surroundings. These can be caused by high electrical consumption, excessive use of cars, infrastructures design and planning and type of materials used, and the decrease of the green area. Due to its presence in different cities of the world and analyzed with relevant studies, it is decided to conduct a study on the main roads of Panama City. It is proposed to investigate the existence of heat islands and their possible causes, due to their unplanned urban development. Four study areas are delimited to collect temperature data with a thermo hygrometer at a time of 8:00 am, 2:00 pm and 8:00 pm in the transition season, rainy and dry. Consequently, isothermal maps were prepared using the EBK method. As a result, differences in maximum and minimum temperatures of $9^{\circ} \mathrm{C}$ for the morning period, $8.1^{\circ} \mathrm{C}$ for the afternoon and $2.4^{\circ} \mathrm{C}$ for the night for the transition season were obtained; values of $10.5^{\circ} \mathrm{C}$ for the morning, $8.1^{\circ} \mathrm{C}$ for the afternoon and $3.3^{\circ} \mathrm{C}$ for the night in the rainy season; and finally the dry season with $7.8^{\circ} \mathrm{C}$ for the morning, $9^{\circ} \mathrm{C}$ for the evening and $2.6^{\circ} \mathrm{C}$ for the night. Heat islands are confirmed and their relationship with tall buildings, car use, the use of asphalt as material for the streets, and environmental factors such as rain, clouds and winds promote the generation of them.

Keywords-Urban heat island, temperature, Panama City, Empirical Bayesian Kriging.

\section{Introducción}

El incremento de la población se ha dado de manera acelerada en los últimos años, promoviendo el nacimiento y sobrepoblación en grandes ciudades a nivel mundial [1]. El desarrollo de las ciudades conlleva la generación de nuevas edificaciones, sean por el ámbito civil, institucional e industrial, carreteras y el uso de medios de transporte. Varios autores han estudiado, desde 1958 la existencia del fenómeno islas de calor urbanas. Se han desarrollado una gran cantidad de observaciones y estudios afirmando su existencia a nivel mundial, sin tener la excepción del tamaño, ubicación sea

Citación: M. Candanedo y D. Villarreal, "Efecto de las islas de calor urbano en las principales vías de la Ciudad de Panamá", Revista de I+D Tecnológico, vol. 16 , no. 2 , pp. (no modificar), 2020

Tipo de artículo: Original. Recibido: 23 de agosto de 2019. Recibido con correcciones: 28 de agosto de 2019. Aceptado: 7 de julio de 2020

DOI.

Copyright: 2020 M. Candanedo y D. Villarreal. This is an open access article under the CC BY-NC-SA 4.0 license (https://creativecommons.org/licenses/by-nc$\mathrm{sa} / 4.0 /)$. 
costera o en tierra y posición geográfica de las ciudades [2]. El fenómeno de islas de calor urbano se define como la diferencia de temperatura entre la zona urbana a comparación de sus alrededores, encontrándose en las áreas más pobladas de la ciudad [3]. El aumento de temperatura provoca una mayor retención de gases de efecto invernadero en las zonas urbana, puesto que la concentración de estos gases ha aumentado de manera alarmante en los últimos años y así, el efecto de islas de calor urbanas contribuye al cambio climático.

\subsection{Principales causas y consecuencias del efecto islas de calor urbanas}

La generación de las islas de calor urbanas proviene del calor antropogénico, geometría urbana y la selección de tipos de materiales de construcción. Estas actividades pueden llegar a tener impactos en el aumento de consumo de energía, aumentar la contaminación del aire y gases de efecto invernadero, comprometer el confort y salud humana y la calidad del agua [4].

\subsubsection{Calor antropogénico}

El consumo de energía eléctrica y el uso de automóviles son elevados por la cantidad de actividades realizadas en la ciudad [5]. Estas actividades utilizan como materia prima los combustibles fósiles que, al ser quemados, en su determinado proceso, liberan cierta cantidad de gases de efecto invernadero, mismos que se quedan atrapados en las zonas urbanas.

\subsubsection{Geometría urbana}

El diseño y planificación para el desarrollo de las ciudades incluye la geometría del diseño de calles, orientación, ejes de calles y altura de los edificios, factor de vista y escala de barriadas locales. Todos estos factores afectan variables ambientales como el flujo de radiación solar y dirección y velocidad de vientos[6]. Los patrones de sombras e intercambio de flujos de calor de las edificaciones son alterados por la geometría urbana modificando las variables ambientales mencionadas.

\subsubsection{Tipos de materiales de construcción}

Los materiales suelen clasificarse por características termales como lo son: calor especifico, color, textura y porcentaje de albedo [7]. Al seleccionar materiales que presenten colores oscuros y un albedo bajo conjunto una reducción de área verde, colaboran a la retención de temperatura en el área. Esto se debe a que estos materiales absorben y retienen mayor calor y las zonas verdes (zona de vegetación) absorben y transforman la luz solar en su proceso de fotosíntesis.

\subsection{Problemática en la Ciudad de Panamá}

La Ciudad de Panamá es la capital de la provincia y República de Panamá, fundada el 15 de agosto de 1519. Según el censo del 2010, consta de unos 430,299 habitantes en el distrito [8]. Localizada en la zona costera del océano Pacífico y cerca del Canal de Panamá. Siendo esta el área de mayor desarrollo urbano a nivel nacional por poseer un gran porcentaje de actividad económica e industrial, y de movilidad urbana. Se demuestra por el plan distrital de la Ciudad de Panamá, la cantidad de empleos es indirectamente proporcional a la cantidad de personas que residen en el área con valores aproximados de 395 mil personas a 135 mil viviendas. Hecho este que sustenta la gran movilidad desde las áreas este y oeste afuera de la ciudad hacia los sitios de empleo. La movilidad urbana puede llegar a ser una posible consecuencia de la generación de islas de calor en la ciudad, ya que los ciudadanos, al vivir en lugares distantes deben movilizarse utilizando vehículos, propios o de transporte público, que generan un $78 \%$ de hidrocarburos y $98 \%$ de monóxido de carbono [9], siendo estos gases de efecto invernadero los que permanecen en el área y aumentan la temperatura del sector. De igual forma, esta consecuencia se da gracias al desarrollo del área y se posiblemente se puede atribuir el modo de planificación de un área urbana es la base de generación del fenómeno descrito.

\subsubsection{Planificación de la Ciudad de Panamá}

El desarrollo de la Ciudad de Panamá se ha dado gracias al impulso del comercio exterior, desde los inicios del descubrimiento del país se promovió una ruta necesaria de transporte y riquezas al nuevo continente. Esto desencadenó a través de los años la expansión de la zona donde los extranjeros definían espacios por sectores de altos y bajos recursos. En los años 60, se presenta un aumento masivo de migración de personas del interior hacia la ciudad por motivos de industrialización y la incursión en el campo generando la búsqueda de nuevos empleos. Trajo como consecuencia a la capital del país un crecimiento espontaneo y toma de tierras no vigilada para la construcción[10].

\subsubsection{Legislación Panameña}

La creación de leyes sobre planificación urbana inició en los años 90. Se incluye tema de ordenamiento 
territorial para el desarrollo urbano, normas de diseño relativas al régimen de propiedad horizontal y reglamento nacional de urbanización del territorio de la República de Panamá, sin embargo, no cuenta con límites permisibles de cambios de zonificación o para disposición de uso de suelos y ausencia de una normativa ambiental obligatoria sobre materiales de construcción. Los materiales cumplen con normas DGNTI-COPANIT basados por resistencias y análisis estructural.

\subsubsection{Planes de Desarrollo Urbano}

De acuerdo con el Tratado Torrijos-Carter, se creó el Plan de Desarrollo Urbano de las Áreas Metropolitanas de Panamá Atlántico - Pacífico elaborado en 1997 con un diagnóstico de cómo se encuentra la ciudad en parte de infraestructuras, viviendas, población, uso de suelos, entre otros. Además de las estrategias para mitigar los problemas de esa época y su debida actualización realizada en el año 2016. No obstante, este plan no tiene un carácter legalmente vinculante y está dirigido de manera general necesitando el apoyo de entidades municipales, la comunidad y los sectores interesados en la parte de planificación para la realización de las ideas propuestas.

Actualmente, la dirección de planificación urbana ha elaborado los planes parciales de ordenamiento territorial (PPOT) para el corregimiento de San Francisco, el plan distrital de Panamá (PDP) incluye un plan estratégico participativo, políticas territoriales, pacto local y un plan local de ordenamiento territorial. Con ello se especifica un pre diagnostico enfocado en las áreas de:

- Ambiente

- Medio rural

- Vulnerabilidad y riesgo

- Patrimonio cultural y arqueológico

- Socioeconómico

- Movilidad

- Transporte y logística

- Suelo urbano y dinámica espacial

- Infraestructura urbana

- Eje transversal, marco legal, políticas, estrategias y normas vigentes

Al desarrollar cada una de las variables, se elaboran ciertas soluciones de mayor importancia a los temas de fomento y repartición equitativa de áreas verdes, reforestación, unión de parques naturales, descentralización y creación de un área compacta de alta densidad y construcción de viviendas asequible. Sin embargo, los planes no cuentan con la idea de regulación de materiales de construcción o iniciativas para realizar una vida sostenible para las personas.

Para el ámbito ambiental se cuenta con la elaboración en conjunto del Banco Interamericano de Desarrollo (BID) y el Municipio de Panamá en 2019 de un Plan de Acción sobre Iniciativa de Ciudades emergentes y sostenibles. Luego de realizar un estudio pertinente a distintos factores dio como resultado los siguientes temas prioritarios: movilidad y transporte, gestión de residuos sólidos, agua, desigualdad urbana y vulnerabilidad ante desastres naturales. Estos son agrupados en tres líneas estratégicas, siendo planificación urbana sostenible, servicios urbanos de calidad, y gestión moderna y eficaz. La parte de mayor importancia en la mitigación de islas de calor, si se presentan en la Ciudad de Panamá, se establece en la clasificación de la primera línea estratégica. Esta habla de establecer transporte de calidad provocando la mitigación de la principal causa de generación de gases de efecto invernadero que es ocasionado por los automóviles. Adicionalmente, la confección de planes estratégicos distritales y locales de ordenamiento territorial ayudan a la mitigación de ellos (cabe mencionar la elaboración de estos en el municipio de Panamá).

Al analizar estas iniciativas, la mejoría en el ámbito de planificación urbana está iniciando y requiere plasmar estas acciones de manera concreta de la mano de la sociedad y los gobernantes.

\section{Metodología}

Para determinar el fenómeno de islas de calor en la Ciudad de Panamá se recolectan datos in situ para confeccionar mapas de isotermas y analizar el su comportamiento. Para ello se debe seleccionar un área de estudio, recolectar datos de temperatura y generar los mapas con el uso del programa ArcGIS.

\section{1 Área de Estudio}

Se selecciona la Ciudad de Panamá para realizar el estudio de islas de calor urbanas por ser el área más desarrollada de la República de Panamá. Para cada zona se establecerá puntos a cada 500 metros para realizar la medición de temperatura se puede apreciar en la figura 1. 


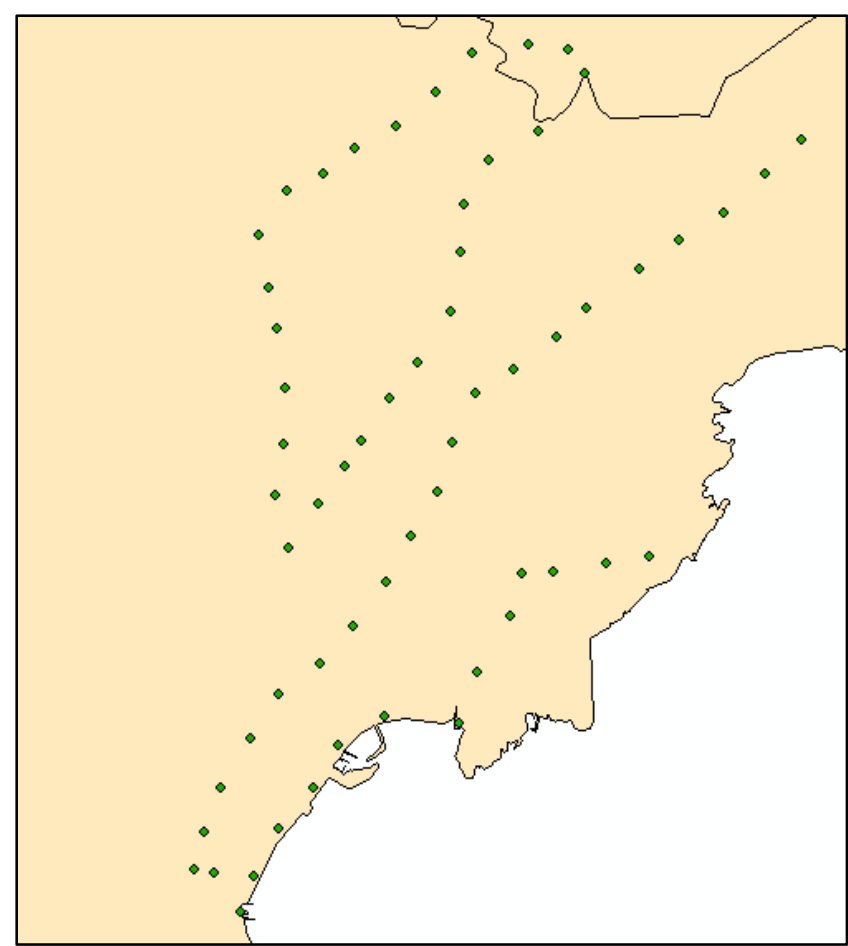

Figura 1. Área de Estudio.

En la tabla 1 se mencionan cada zona, su longitud, y una descripción de los puntos iniciales y finales de cada tramo.

Tabla 1. Zonas de Estudio

\begin{tabular}{|c|c|c|c|c|}
\hline Zona & Vías & $\begin{array}{c}\text { Longitu } \\
\mathbf{d}(\mathbf{k m})\end{array}$ & $\begin{array}{c}\text { Inicio de } \\
\text { Tramo }\end{array}$ & $\begin{array}{c}\text { Final del } \\
\text { Tramo }\end{array}$ \\
\hline $\begin{array}{c}\text { Zona } \\
1\end{array}$ & $\begin{array}{c}\text { Vía Ricardo J. } \\
\text { Alfaro / } \\
\text { Tumba } \\
\text { Muerto }\end{array}$ & 6,97 & $\begin{array}{c}\text { Hospital } \\
\text { San Miguel } \\
\text { Arcángel }\end{array}$ & $\begin{array}{c}\text { Cervecería } \\
\text { Nacional }\end{array}$ \\
\hline $\begin{array}{c}\text { Zona } \\
2\end{array}$ & $\begin{array}{c}\text { Vía Simón } \\
\text { Bolívar/ } \\
\text { Transístmica }\end{array}$ & 5,69 & $\begin{array}{c}\text { Puente } \\
\text { frente a } \\
\text { Cervecería } \\
\text { Nacional }\end{array}$ & $\begin{array}{c}\text { Estación } \\
\text { San } \\
\text { Miguelito }\end{array}$ \\
\hline $\begin{array}{c}\text { Zona } \\
3\end{array}$ & $\begin{array}{c}\text { Vía España - } \\
\text { Calidonia }\end{array}$ & 9,33 & $\begin{array}{c}\text { McDonald's } \\
\text { de Balboa }\end{array}$ & $\begin{array}{c}\text { Plaza 5 de } \\
\text { mayo }\end{array}$ \\
\hline $\begin{array}{c}\text { Zona } \\
4\end{array}$ & $\begin{array}{c}\text { Cinta Costera } \\
- \text { Vía Israel }\end{array}$ & 6,6 & $\begin{array}{c}\text { Rotonda del } \\
\text { Mercado de } \\
\text { Mariscos }\end{array}$ & $\begin{array}{c}\text { Hotel } \\
\text { Sheraton }\end{array}$ \\
\hline
\end{tabular}

Al ubicar los tipos de zonificaciones de las zonas a evaluar, se determina que las zonas 1, 2, 3 y parcialmente la 4 tienen en su mayoría la clasificación de residencial de alta densidad y comercial de intensidad alta (RM3C2), puede ser oficinas, locales, instituciones, multifamiliares, entre otros. La parte final de la zona 4 está contemplada por comercios de alta intensidad (C2) [11].

Otro punto para destacar es el material utilizado para la confección de carreteras en la Ciudad de Panamá, en su mayoría está constituida por asfalto y otra por concreto. Además de la construcción de carreteras, se realizan rellenos con asfalto tipo cartera por grietas que suelen salir en la carretera. Se debe mencionar como el asfalto es un contaminante debido a su alta absorción de calor debido a su bajo albedo [12].

\subsection{Recolección de Datos}

El estudio se desarrolla mediante la medición de temperatura en las principales vías de la Ciudad de Panamá. Para las vías delimitadas se establecerá puntos específicos a cada 500 metros medidos, la longitud y posición geográfica de cada vía fue determinada por el programa Google Earth. El recorrido se realizará en automóvil y se utilizará un termo-higrómetro calibrado $+/-1{ }^{\circ} \mathrm{C}$ para medir temperatura. Los instrumentos de medición se deben colocar sin manipulación alguna y debe esperar un mínimo de dos minutos para tener un valor estable.

Panamá consta de dos estaciones: seca y lluviosa, donde la seca se establece por cuatros meses y la lluviosa por ochos meses del año. El intervalo de tiempo puede ser relativo, puesto puede surgir fenómenos del niño o la niña en el país. Por ello, las fechas de mediciones se basaron en las estaciones climatológicas del país, donde se efectuará tres mediciones en los 12 meses. Su división será en temporada seca en el mes de enero del 2019, transición de temporada seca a lluviosa en mayo del 2018 y la temporada lluviosa con fecha de agosto del 2018. Los datos térmicos se recolectarán en tres intervalos del día: matutino, vespertino y nocturno. Las horas de inicio de cada medición son 8:00 am, 2:00 pm y 8:00 pm.

\subsection{Creación de Mapas de Isotermas}

Las isotermas serán confeccionadas por el software ArcGIS, específicamente con los programas Arcmap. Los pasos para la manipulación de los datos, observación de comportamiento de temperatura y elaboración de mapas de isotermas mediante un modelo no lineal.

Es importante resaltar que los datos seleccionados para realizar el estudio de las islas de calor es la temperatura. Esta variable tiene un comportamiento no lineal, puesto que depende de distintos parámetros climáticos [13] que no son el objetivo del presente 
estudio. Esto concede que la creación de Isotermas, los triangulared irregular network (TIN) y Rasters no demuestran un comportamiento real, debido a que estas formas de representaciones digitales son adecuadas para un comportamiento lineal. Las exhibiciones de las representaciones se utilizan para ver un comportamiento en general. Para una representación más conveniente, se debe utilizar un método de interpolación no lineal que cumpla con las especificaciones descritas para este estudio. Esto será explicado en el a continuación.

Para determinar un comportamiento y la existencia de Islas de Calor se procede a escoger un método de interpolación con los datos de temperatura, es decir, los puntos transformados en ArcGIS. Su selección está basada en la lección de Eric Krause, "Analizar el calentamiento urbano mediante kriging. Encuentre islas de calor y poblaciones en riesgo en Madison, Wisconsin.". El programa cuenta con una las herramientas de análisis geo-estadístico donde presenta varios métodos de interpolación. Uno de ellos es el Empirical Bayesian Kriging que fue seleccionado para este estudio.

Empirical Bayesian Kriging (EBK) es un modelo actualizado del Kriging simple, siendo más fácil y preciso de usar. Una de las principales características del método de interpolación Kigring simple es que utiliza un solo semivariograma donde infiere que este fue diseñado a la perfección y cualquier valor que no siga la tendencia será excluido del mismo, mientras el EBK crea semivariogramas por cada subconjunto de datos para realizar distintas simulaciones que son utilizadas para crear nuevos subconjuntos y combinarse entre sí para obtener un mapa de predicción. Siendo así una forma más precisa y real de la interpolación [14].

\section{Análisis de Resultados}

La recolección de datos de temperatura en las cuatro distintas zonas, que incluyen la Vía Ricardo J. Alfaro/Tumba Muerto, Vía Simón Bolívar/Transístmica, Vía España y Cinta Costera, de la Ciudad de Panamá mostrada en la figura 2 han dado como resultado una diferencia de valores máximos y mínimos por cada temporada y horario que se muestra en la tabla 2.

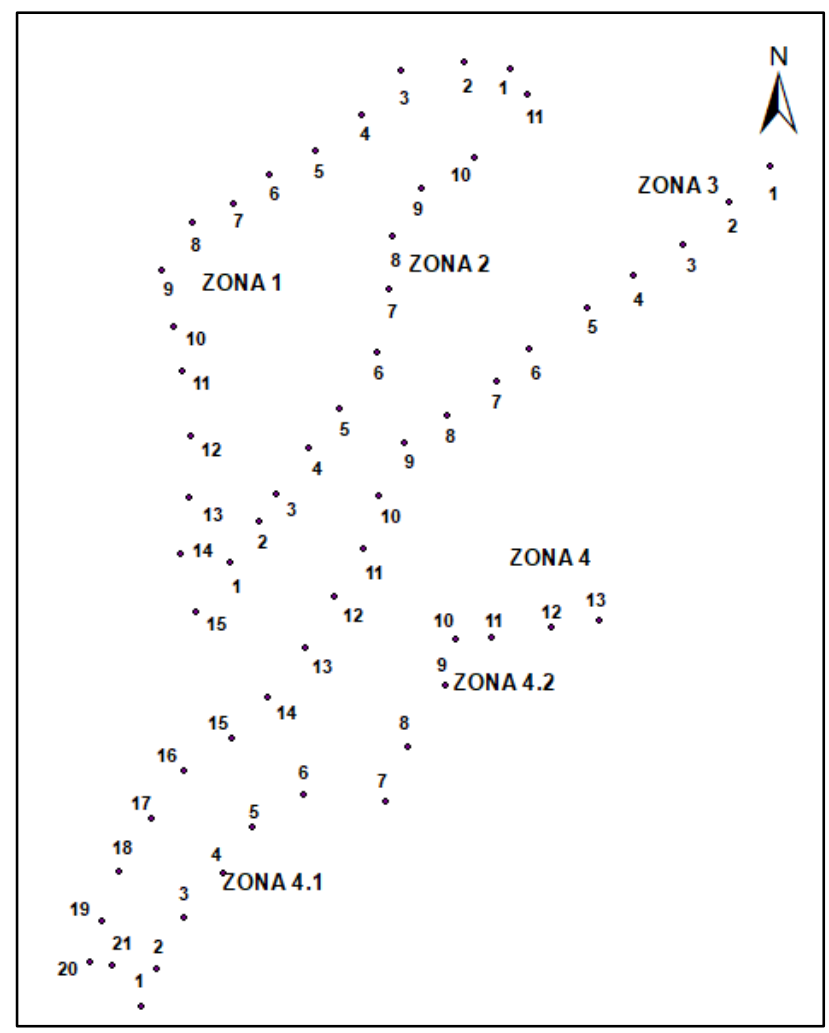

Figura 2. Zonas de Estudio.

Tabla 2. Diferencia de Temperaturas

\begin{tabular}{|c|c|c|c|}
\hline Temporada & Matutino & Vespertino & Nocturno \\
\hline $\begin{array}{c}\text { Transición: } \\
\text { Seca a Lluviosa }\end{array}$ & $9^{\circ} \mathrm{C}$ & $8.1^{\circ} \mathrm{C}$ & $2.4^{\circ} \mathrm{C}$ \\
\hline Lluviosa & $10.5^{\circ} \mathrm{C}$ & $8.1^{\circ} \mathrm{C}$ & $3.3^{\circ} \mathrm{C}$ \\
\hline Seca & $7.8^{\circ} \mathrm{C}$ & $9^{\circ} \mathrm{C}$ & $2.6^{\circ} \mathrm{C}$ \\
\hline \multicolumn{4}{|r}{}
\end{tabular}

La tabla 2 demuestra como la temporada lluviosa mantiene la mayor dispersión de temperatura para el horario matutino con $10.5^{\circ} \mathrm{C}$ mientras que la temporada seca tiene la menor de $7.8^{\circ} \mathrm{C}$. Por otro lado, para el horario vespertino se permanece la misma diferencia para la temporada de transición y lluviosa de $8.1^{\circ} \mathrm{C}$ mientras que la seca mantiene la $\mathrm{m}$ mayor diferencia con $9^{\circ} \mathrm{C}$. Por último, el horario nocturno tiene $3.3^{\circ} \mathrm{C}$ de mayor diferencia para la temporada lluviosa, un $2.6^{\circ} \mathrm{C}$ para la seca y $2.4^{\circ} \mathrm{C}$ para transición de seca a lluviosa. Estos datos indican la presencia del fenómeno de islas de calor urbano, puestos manifiestan gratas diferencias de temperaturas en el área.

En segunda instancia, se observa la interpretación de los mapas de isotermas elaborados con el método de interpolación Empirical Bayesian Krigging (EBK). El análisis de los mapas de isotermas mostrará el 
desplazamiento de las islas de calor y una breve caracterización sobre posibles causas de estas.

Se debe recordar que el análisis específicamente del método EBK se debe al comportamiento de la temperatura, descrita como no lineal. Siendo establecido por Eric Krause, el método más apropiado para realizar esta interpolación utilizando el programa ArcGIS.

Se resalta que los datos de temperatura obtenidos pueden varias con los datos resultantes de interpolación. Se debe a que, al momento de efectuar la interpolación, este interpola con todos los valores cercanos a la superficie a estudiar dando como resultado un rango de error desde el valor que se utilizó como control al resultado obtenido.

El resultado de la interpolación consta de nueve distintos mapas de isotermas:

- Horario Matutino: temporada seca, transición y lluviosa

- Horario Vespertino: temporada seca, transición y lluviosa

- Horario Nocturno: temporada seca, transición y lluviosa

\subsection{Descripción de mapas de isotermas}

Se procederá a realizar la descripción de cada mapa dividido por temporada y medición diaria. Se procede a realizar una comparación descriptiva sobre el período de medición diaria, esto será de la siguiente forma: matutino, vespertino y nocturno.

\subsubsection{Horario Matutino}

Al visualizar las figuras 3, 4 y 5 de los mapas sobre temporada de transición: seca a lluviosa, lluviosa y seca en horario de la mañana con inicio a las 8:00 a.m. han prevalecido las siguientes características notorias tales como:

Los cuatro primeros puntos localizados desde la entrada a la vía transístmica hasta los estacionamientos del Banco Nacional de Panamá ubicados en la zona 2, muestran un comportamiento de mantener una temperatura más baja en las tres temporadas. Los promedios de las temperaturas son de $29^{\circ} \mathrm{C}$ para la temporada seca, $27.8^{\circ} \mathrm{C}$ para la temporada de transición y $27.5^{\circ} \mathrm{C}$ para la temporada lluviosa.

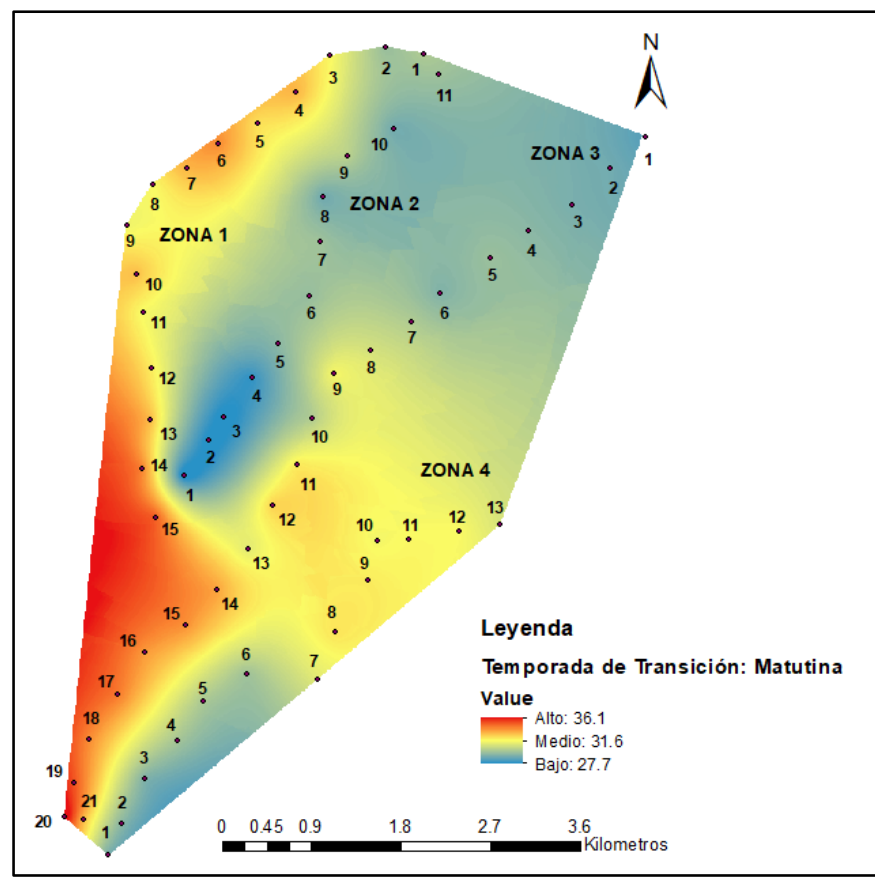

Figura 3. Mapa de Isotermas modelo EBK - Temporada de Transición: Matutina.

La primera zona ubicada en la vía Ricardo J. Alfaro se mantiene como la zona de mayor concentración de temperatura para las temporadas de transición seca a lluviosa y lluviosa. Un punto a destacar es el encontrado en los estacionamientos del supermercado Xtra (punto 5) de $36.4{ }^{\circ} \mathrm{C}$, siendo el valor más alto del mapa de Isoterma y de la Temporada lluviosa. Otra característica para mostrar, son sus variaciones de temperatura en las tres distintas temporadas como lo son: para temporada seca se tiene una distribución variada de temperatura a lo largo de toda la vía desde la parada de bus del Hospital San Miguel Arcángel (punto 1) hasta la estación de gasolinera Delta en frente de la Cervecería Nacional (punto 15) y una diferencia de valor máximo y mínimo de $4^{\circ} \mathrm{C}$, en la temporada de transición sus valores máximos se encuentran a final de la vía y un rango de temperatura de $4.8^{\circ} \mathrm{C}$ y en la temporada lluviosa la concentración de temperatura altas esta al inicio del tramo y su rango de temperaturas es de $5.8^{\circ} \mathrm{C}$. 


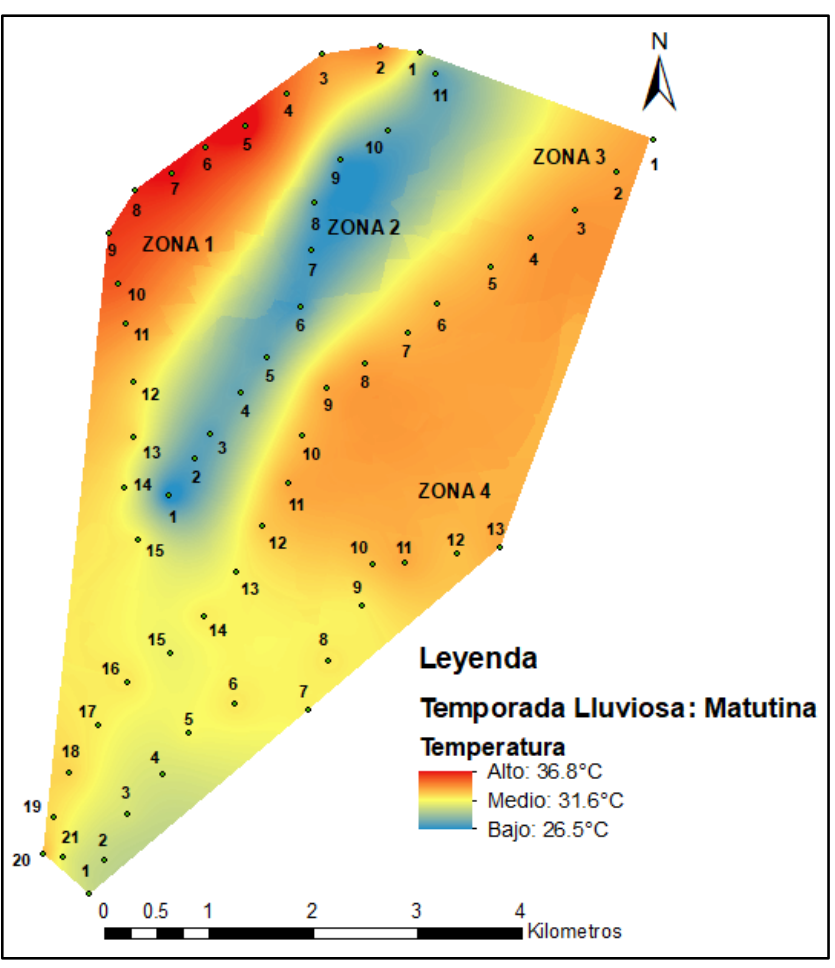

Figura 4 Mapa de Isotermas modelo EBK - Temporada Lluviosa: Matutina.

La zona 3 muestra un comportamiento distinto en las tres mediciones de temporadas. Se muestra un aumento de temperatura de $28.5^{\circ} \mathrm{C}$ hasta $36.5^{\circ} \mathrm{C}$ dando una diferencia de $8^{\circ} \mathrm{C}$ en la temporada de transición. Se tiene excepciones como la isleta frente a los bomberos de Vía España (punto 10) y Bellini en Vía España (punto 13) que no siguen el patrón. Igualmente, al finalizar la Vía España, el aumento de temperatura es extremo puesto que llega a $36.5^{\circ} \mathrm{C}$ en la plaza 5 de mayo. Para la temporada lluviosa, se ve una disminución de temperatura desde inicio hasta el final, por motivo que se encontraba nublado la vía y su diferencia de valores consiste en $2.4^{\circ} \mathrm{C}$. Mientras que el comportamiento de la vía España en la temporada seca, manifiesta una concentración de temperatura en los extremos, el punto 4 ubicado en el comercio Tasco Battery cuenta con la temperatura de $35.8^{\circ} \mathrm{C}$ como el valor más alto, no obstante, al complementar con las observaciones tomadas en campo al momento de realizar la medición se estableció una gran cantidad de vehículos pasando por este punto. Igualmente, el punto localizado en la estación 5 de mayo cuenta con la misma temperatura. El rango estipulado por toda la vía es de 32.6 a $33.8^{\circ} \mathrm{C}$ donde cinco puntos salen de estos valores, ellos son los dos de mayor concentración de temperatura y los otros tres son por factores de sombra de edificios (punto 7 - especificado como los estacionamientos del comercio Friolín), mientras que los puntos restantes son influenciados por brisas fuertes y cierta nubosidad que son mostrados en el mapa. Se debe mencionar que los puntos afectados son los que tienen un rango de temperatura de $30^{\circ} \mathrm{C}$ a $32.6^{\circ} \mathrm{C}$.

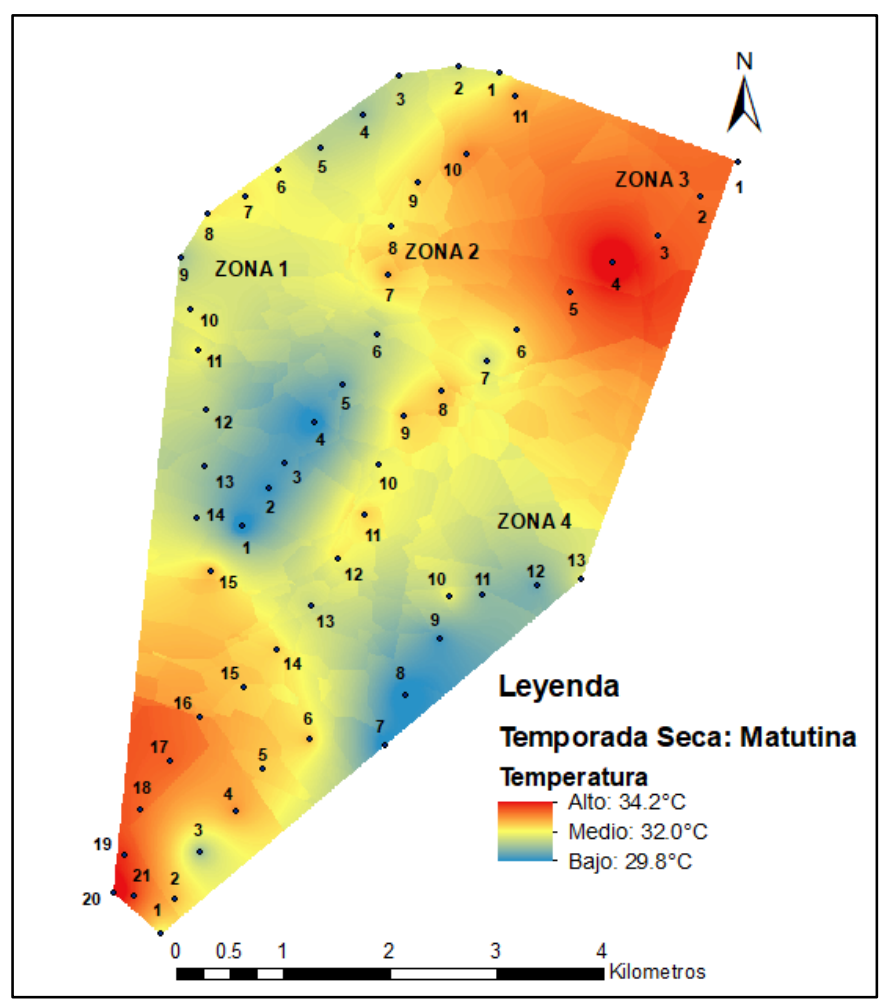

Figura 5. Mapa de Isotermas modelo EBK - Temporada Seca: Matutina.

Para la última zona, se observa la división de temperaturas desde el inicio al final de la cinta costera (punto 1 al punto 6) y desde el parque de cercano a la cinta costera hasta el hotel Sheraton (punto 7 al punto 13). Estas serán clasificadas para mejor comprensión como zona 4.1 y zona 4.2 como se muestra en la ilustración 29. Para las temporadas de transición y lluviosa se muestra una zona 4.1 con menores valores de temperatura (diferencia de valores máximos y mínimos fueron de $29.5^{\circ} \mathrm{C}<31.5^{\circ} \mathrm{C}$ y $31^{\circ} \mathrm{C}<32.5^{\circ} \mathrm{C}$ respectivamente) a diferencia de la temporada seca que muestra los menores valores en la zona 4,2 rango de valores de $31,8^{\circ} \mathrm{C}>30,5^{\circ} \mathrm{C}$. Se debe mencionar que los datos adquiridos en la temporada seca de la zona 4.2 son influenciados por las sombras de los edificios a excepción en la parada de bus del centro comercial Multiplaza y la esquina del Hotel Sheraton. 


\subsubsection{Horario Vespertino}

La primera zona cuenta con datos solamente para la temporada seca y transición, puesto que para la lluviosa no se pudo recolectar datos a causa de lluvia, referirse a la figura 6,7 y 8 para observar su comportamiento en los mapas de isotermas. Al realizar un análisis general se muestra en transición un rango de temperatura de $2.9^{\circ} \mathrm{C}$, siendo la parte superior a excepción de los dos primeros puntos (parada de bus en el Hospital San Miguel Arcángel y los estacionamientos de Artec) los valores más bajos. Para la temporada seca la diferencia de temperatura es de $4.7^{\circ} \mathrm{C}$, donde sus valores máximos están distribuidos de manera aleatoria por la vía.

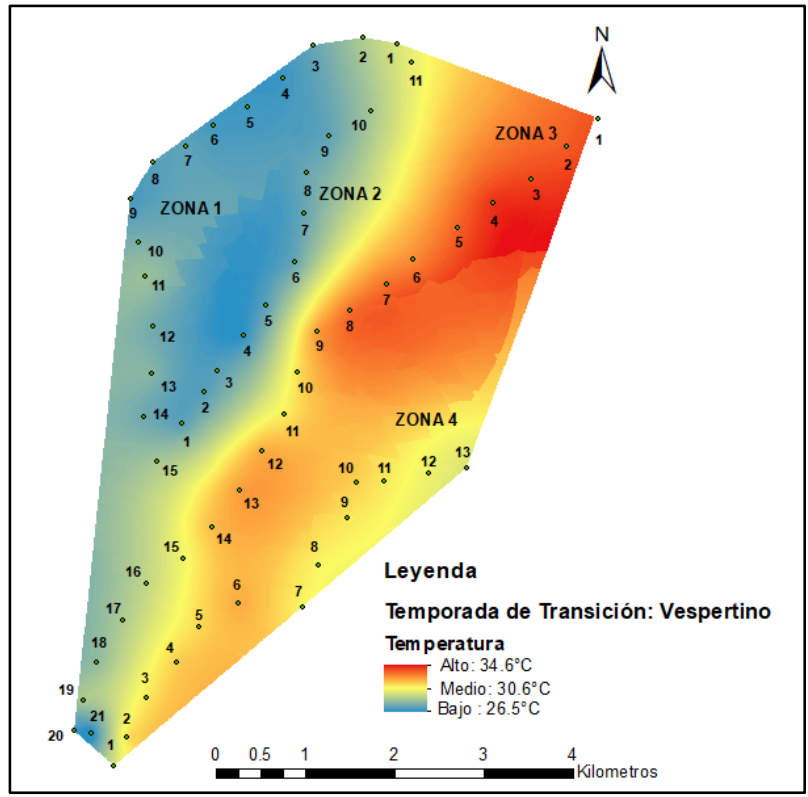

Figura 6. Mapa de Isotermas modelo EBK - Temporada de Transición: Vespertino.

La zona 2 cuenta con un comportamiento muy peculiar en esta clasificación. Debido a que en la temporada seca se muestran los valores más elevados no solo en las otras temperaturas sino en su mapa producto del EBK. Su rango de valores va desde $35.2^{\circ} \mathrm{C}$ en la isleta frente a la estación del metro de San Miguelito (punto 11) hasta $39.9^{\circ} \mathrm{C}$ en la entrada a la vía Simón Bolívar (punto 1). De manera peculiar la temperatura va incrementando desde el punto 2 la entrada al supermercado Xtra hasta la entrada a la casa de encuentro El Puente como punto 10. Se percibe que el día de su medición contaba con un factor climático puesto que se realizó otra medición en la zona 1 y presentó valores elevados a los de la transístmica. Para la temporada de transición, se muestra un rango de diferencia de $3.5^{\circ} \mathrm{C}$, tomando una tendencia de valores mínimos al inicio de la vía con promedio de $27.2^{\circ} \mathrm{C}$ y van en aumento, se debe mencionar la presencia de lluvia a la mitad del recorrido. Para la temporada faltante, se ve un rango de $3.2^{\circ} \mathrm{C}$ una tendencia distinta al colocarse los valores máximos al inicio de la de vía y disminuir hasta finalizar.

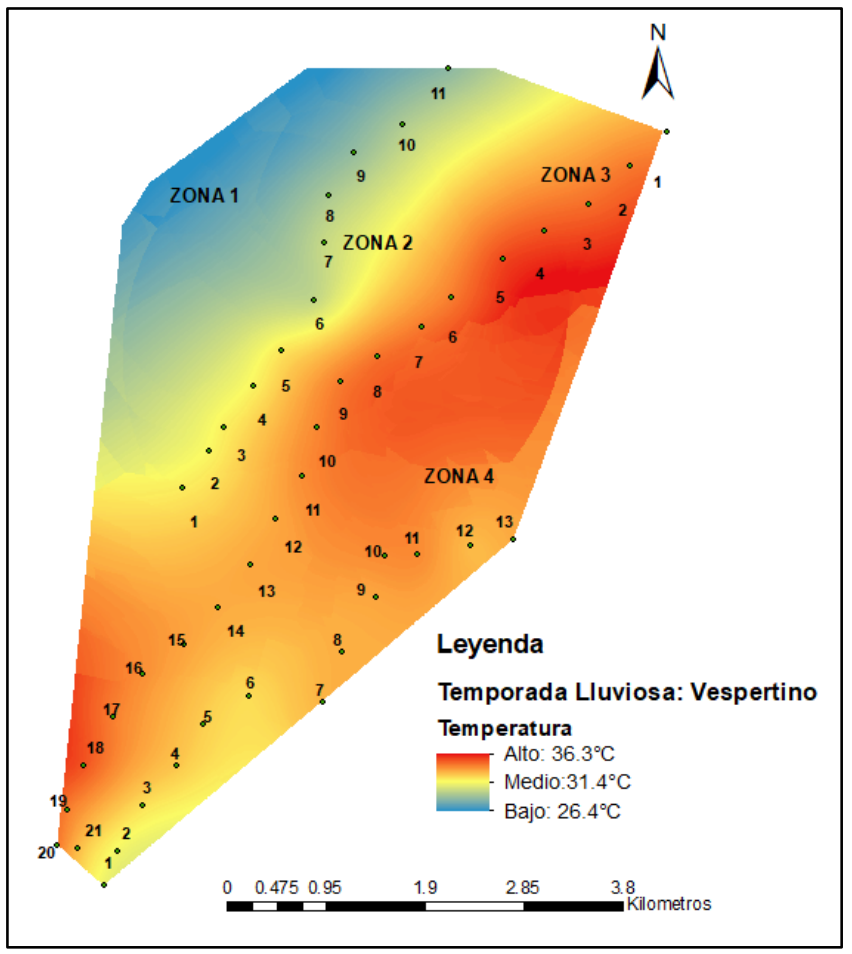

Figura 7. Mapa de Isotermas modelo EBK - Temporada Lluviosa: Vespertino.

La tercera zona muestra en sus tres temporadas un descenso de temperatura desde su inicio de $33.5^{\circ} \mathrm{C}$ a $31.5^{\circ} \mathrm{C}$ en temporada seca, $33.6^{\circ} \mathrm{C}$ a $25.9^{\circ} \mathrm{C}$ en temporada de transición y $33.4^{\circ} \mathrm{C}$ a $34.5^{\circ} \mathrm{C}$ en temporada lluviosa (aunque se ve un aumento en los valores del medio existe variación). Para lluviosa y transición se muestran datos una pequeña variación aleatoria de datos, pero con la tendencia de los valores de mayor temperatura al inicio. Por otra parte, se tienen los rangos de temperatura de lluviosa de $2.9^{\circ} \mathrm{C}$ y transición de $8.9^{\circ} \mathrm{C}$. La temporada seca muestra además su disminución de temperatura a través del mapa que sus datos de menor temperatura tienen la observación del paso de la brisa por el punto al realizar su medición y un rango de temperatura de $3.9^{\circ} \mathrm{C}$. 
La cuarta zona dividida por zona 4.1 y 4.2 demuestra en la temporada lluviosa donde se muestra un rango de valores de $3^{\circ} \mathrm{C}$, donde la zona 4.1 tiene una menor temperatura promedio de $31^{\circ} \mathrm{C}$ a la zona 4.2 con un valor de $33.1^{\circ} \mathrm{C}$. Se destaca la presencia de nubes parciales por todo el recorrido y en su valor máximo de $33.9^{\circ} \mathrm{C}$ localizado en la salida del centro comercial Multiplaza la presencia de tráfico. Mientras que la tendencia en la temporada de transición y seca muestran una variación de datos por toda la vía. Tanto así que sus promedios por vías son los siguientes: para transición zona 4.1 es de $31.1^{\circ} \mathrm{C}$ y zona 4.2 de $30.6^{\circ} \mathrm{C}$ y para temporada seca zona 4.1 con $34.94^{\circ} \mathrm{C}$ y zona 4.2 con $34.99^{\circ} \mathrm{C}$.

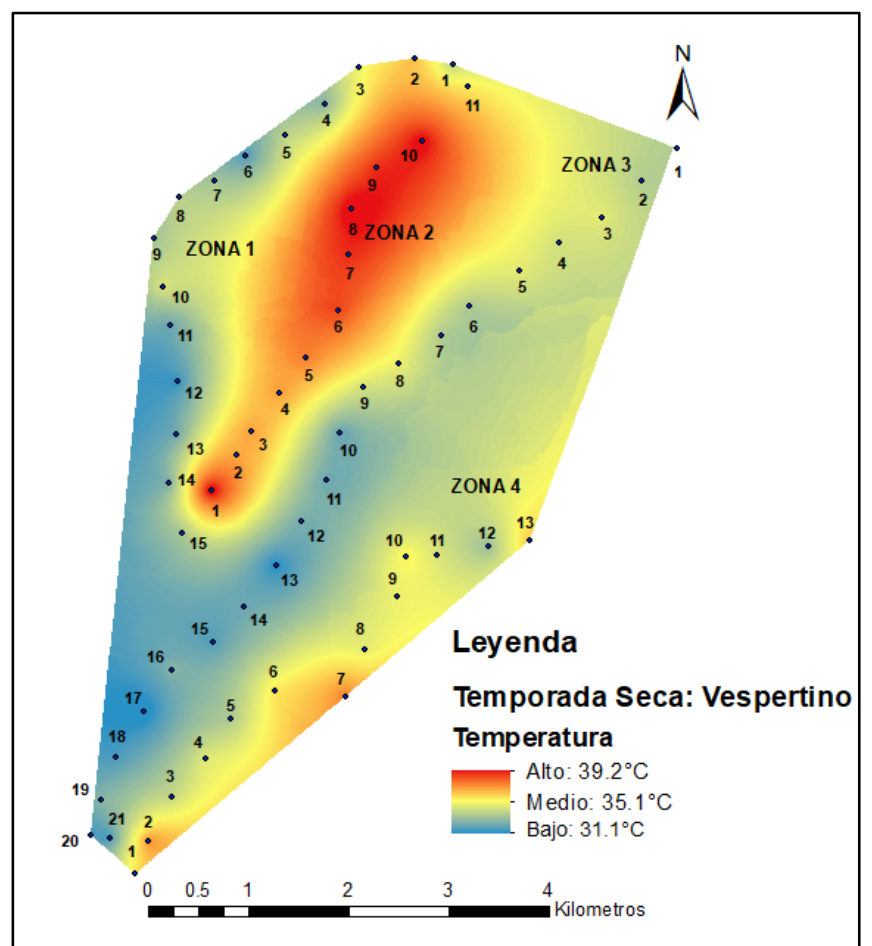

Figura 8. Mapa de Isotermas modelo EBK - Temporada Seca: Vespertino.

\subsubsection{Horario Nocturno}

Para la temporada lluviosa se ve en la primera zona una diferencia de temperatura de $0.7^{\circ} \mathrm{C}$, su comportamiento se ve de esta manera por la lluvia generada en la tarde y un poco llovizna en la noche, como lo muestran las lecturas en los estacionamientos del supermercado Xtra (punto 5). No obstante, este punto adquiere un valor de $27.6^{\circ} \mathrm{C}$ mientras está el evento climático de la llovizna. Su punto de mínimo valor es la isleta frente al puente del trébol cercano a la casa de los materiales (punto 7 ) con $27.1^{\circ} \mathrm{C}$ y el más alto en la parada de bus de la USMA (punto 4) con $27.8^{\circ} \mathrm{C}$. Para la temporada seca, aunque se ve una alta concentración de temperaturas al inicio de la vía, se contempla una variación de datos en los puntos consiguientes. Se observan los valores máximos es de $28.1^{\circ} \mathrm{C}$ en los estacionamientos de la Clínica Yee frente a la UIP y su valor mínimo de $26.4^{\circ} \mathrm{C}$ en la esquina del puente elevado que une la parada de bus La Locería con Plaza Edison. Por último, la temporada de transición muestra dispersiones en los puntos con un rango de temperatura de $1.2^{\circ} \mathrm{C}$.

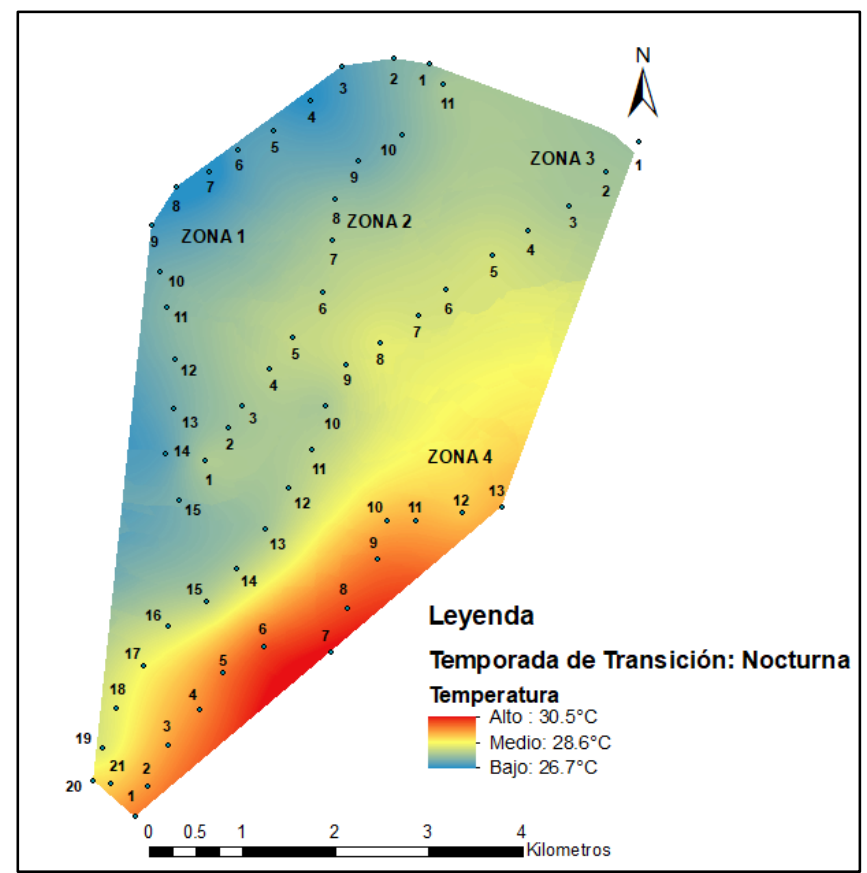

Figura 9. Mapa de Isotermas modelo EBK - Temporada de Transición: Nocturna.

La zona 2 contempla distintas variaciones en las tres temporadas del año descritas en el estudio, ver figura 9, 10 y 11 . Al tener un comportamiento disperso de valores en la temporada de transición, su diferencia de valores está en $1.3^{\circ} \mathrm{C}$ con su valor máximo en el punto 1 , en la entrada a la vía transístmica y su valor mínimo en la parada de la estación del metro Pueblo Nuevo. Para la temporada seca se observa una disminución de temperatura desde su inicio al final de la vía, sin embargo, los valores mínimos tomados en los puntos finales de la vía se ven afectados por la brisa que se ve en las observaciones. Las diferencias de temperaturas se ven reflejado un valor de $0.5^{\circ} \mathrm{C}$ con valores de $27.5^{\circ} \mathrm{C}$ que se repite en cuatro puntos de la zona (entrada al 
supermercado Xtra, frente al local comercial de Terry Llantas, estación del metro Pueblo Nuevo e isleta frente a la estación del metro San Miguelito). Para la temporada lluviosa se ve un incremento de temperatura desde su inicio al final, los valores tienen una diferencia de $1.3^{\circ} \mathrm{C}$ con su valor mínimo en la entrada a la vía transístmica con $27.4^{\circ} \mathrm{C}$ y el final en la estación de San Miguelito con $28.8^{\circ} \mathrm{C}$.

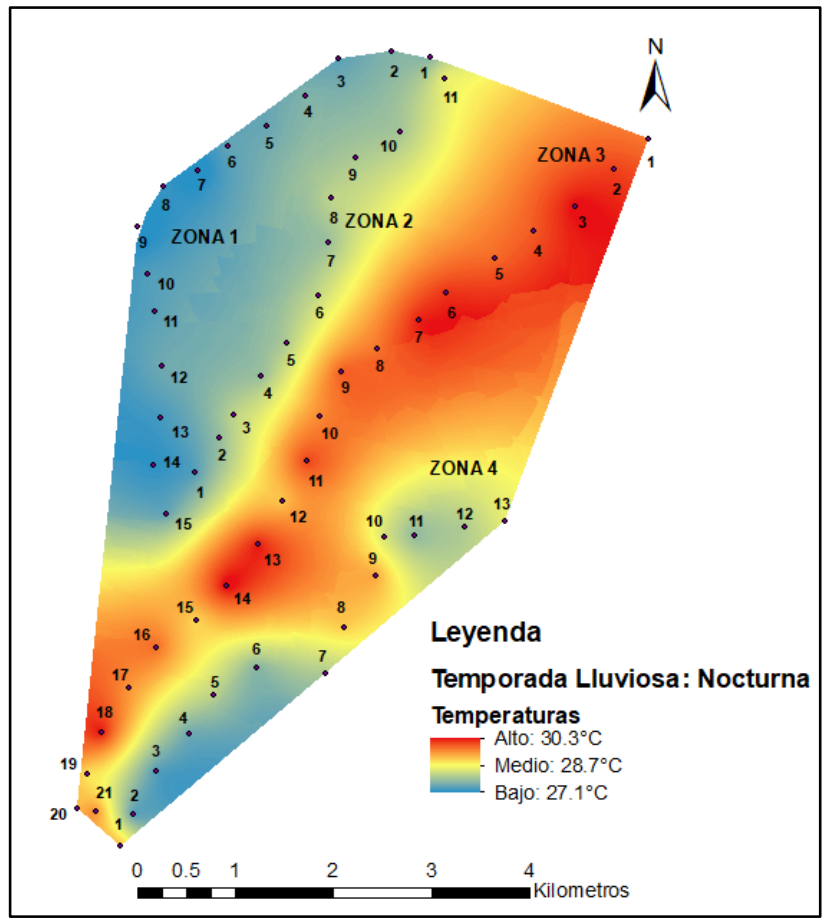

Figura 10. Mapa de Isotermas modelo EBK - Temporada Lluviosa: Nocturna.

La zona 3 comprende datos dispersos en su temporada de transición con una diferencia de valores de temperatura de $1.7^{\circ} \mathrm{C}$ y un promedio de $28.1^{\circ} \mathrm{C}$. La temporada lluviosa muestra datos más dispersos sin embargo en el mapa se nota una distribución de temperaturas altas cercanas al valor máximo del mapa de isotermas. Las diferencias de temperaturas son de $1.7^{\circ} \mathrm{C}$ y un promedio de $29.7^{\circ} \mathrm{C}$. Por último, la temporada seca tiene una diferencia de temperatura en su vía de $2.2^{\circ} \mathrm{C}$, al observar el comportamiento de temperaturas se visualiza una disminución de alternados hasta finalizar la vía.

Por último, la zona 4 demuestra para la temporada de transición los valores más altos de su mapa, esto dividido por valores promedios por zona 4.1 y 4.2 de $29.7^{\circ} \mathrm{C} \mathrm{y}$ $29.6^{\circ} \mathrm{C}$, respectivamente. Para la temporada de invierno, las temperaturas promedias por zonas 4.1 y 4.2 fueron de $27.9^{\circ} \mathrm{C}$ y $28.5^{\circ} \mathrm{C}$ y para la temporada seca de $26.9^{\circ} \mathrm{C}$ y $27.7^{\circ} \mathrm{C}$. Como puntos de interés tenemos los más altos de la zona identificados como la rotonda de la cinta costera 3 frente al mercado de marisco (punto 1), los estacionamientos del McDonald's de Paitilla y la parada de bus de la Escuela Secundaria Profesional Isabel Herrera de Obaldía con valores de 29.1, 29.3 y 29.4 grados Celsius.

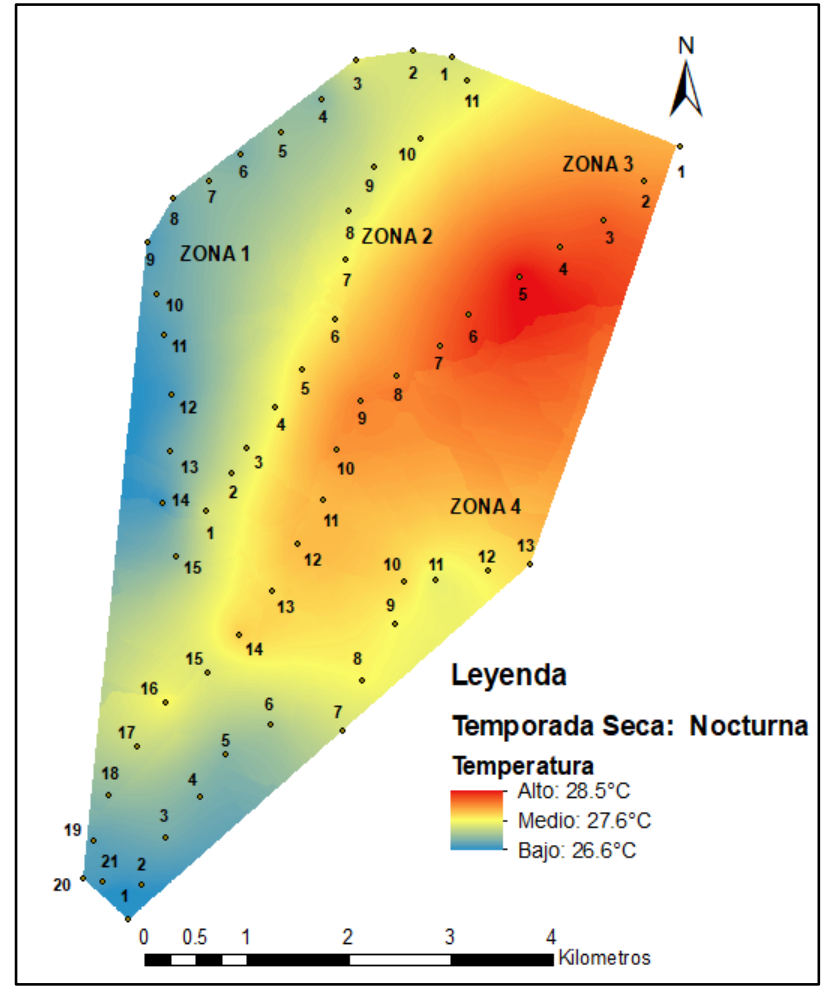

Figura 11. Mapa de Isotermas modelo EBK - Temporada Seca: Nocturna.

\section{Conclusiones}

- Las Islas de Calor se definen por la diferencia de temperatura respecto a un área urbana con sus alrededores, al realizar el análisis correspondiente para evaluar la existencia de estas se determina la presencia de Islas de Calor en las principales vías de la Ciudad de Panamá debido a las distintas concentraciones de temperaturas a lo largo de las vías demostrados no solamente en su horario de recolección sino por su temporada. Aunque se conoce que las temperaturas varían de manera climatológica en distintas horas, días y temporadas del año, las diferencias de valores presentados muestran una dispersión muy elevada en relación a su área estipulada. Para ello se aprecia este comportamiento 
por las diferencias de valores mínimos y máximos encontrados en cada división, por ejemplo: para la temporada de transición seca a lluviosa se muestra los valores de $9^{\circ} \mathrm{C}$ para el período matutino, $8.1^{\circ} \mathrm{C}$ para la tarde y $2.4^{\circ} \mathrm{C}$ para la noche; para la temporada lluviosa los valores de $10.5^{\circ} \mathrm{C}$ para la mañana, $8.1^{\circ} \mathrm{C}$ para la tarde y $3.3^{\circ} \mathrm{C}$ para la noche; y por último la temporada seca con $7.8^{\circ} \mathrm{C}$ para el horario matutino, $9^{\circ} \mathrm{C}$ para el vespertino y $2.6^{\circ} \mathrm{C}$ para el nocturno.

- Al evaluar las diferencias de temperatura de cada punto en cada estación se observa que la temporada lluviosa proporciona los datos más dispersos, por consiguiente, la temporada de transición seca a lluviosa $\mathrm{y}$, por último, la temporada seca. Esto promueve la idea del factor de la lluvia/ciclo del agua puede haber creado las grandes diferencias de temperaturas en las temporadas de transición y mayormente en la lluviosa, y los valores máximos y mínimos para el horario matutino en la temporada lluviosa, puesto una las principales propiedades físicas del agua es su capacidad de absorción de calor. Por otra parte, en la tarde los datos de valores máximos proponen la premisa anterior y por la noche se ve pequeñas variaciones en la tendencia puesto no se obtiene las radiaciones del sol sino otras características climáticas nocturnas. Las contribuciones del trabajo y su grado de relevancia.

- El tráfico vehicular y materiales de construcción se conoce que las carreteras son construidas con la finalidad de proporcionar una vía adecuada para el paso de automóviles, no obstante, ciertos puntos de estudio como Tasco Battery en Río Abajo (punto 4 zona 3) muestra altas temperatura en la temporada seca horario matutino causado posiblemente por el congestionamiento vehicular que se presentó en la recolección de dato en este punto.

Por otra parte, la visualización de los mapas de isotermas del área inicial de la zona 3 ubicada en Río Abajo demuestra los valores más altos de temperaturas, una posible explicación se da por la gran concentración de vehículos siendo una zona de confluencia de arterias vehiculares importantes. Sumado a esta característica, se distingue la presencia extensiva de planchas de asfalto y concreto, y una zona de gran actividad comercial que genera un incremento de número vehículos dentro de la zona.

Los edificios de alta densidad promueven cierto efecto a este fenómeno, se ve demostrado en los puntos de estacionamientos de Friolín (punto 7 - zona), el área de infraestructuras de la cinta costera (zona 4.2) y la entrada de la vía Transístmica (punto 1 - zona 2) desde el puente hasta los estacionamientos del Banco Nacional (punto 4 - zona 2) donde se perciben las características de sombras en ciertas partes de las vías. Esto puede causar el descenso de temperaturas, sin embargo, al ser edificios pueden absorber o reflejar la luz de sol, que puede incrementar su consumo energético, la calidad ambiental interna o elevar las temperaturas a sus alrededores donde no está reflejado la sombra que contribuye a la generación de islas de calor.

- Los factores de nubosidad, viento, lluvia y características climáticas pueden establecer un descenso o aumento de temperatura en las áreas. Como muestras el viento, la lluvia y las nubes ocasionan una disminución de temperatura como se observó en distintos puntos de las tres distintas temporadas, por ejemplo, en la temporada seca horario nocturno el último punto ubicado en la en frente de la estación del metro de San Miguelito punto 11) influenciado por brisa o Bellini en la Vía España (punto 13 - zona 3) horario matutino tiene su descenso de temperatura por nubosidad. El factor de lluvia no facilito la recolección de datos para la temporada lluviosa en horario vespertino para la Vía Ricardo J. Alfaro - zona 1, no obstante, los valores nocturnos no sólo de su zona sino del mapa de isotermas muestra los valores más bajo y se presume este comportamiento por la lluvia dada en horas anteriores. Por otro lado, la zona 2 - Vía Simón Bolívar muestra las temperaturas más elevadas en la temporada seca horario vespertino, esto es uno de los puntos de mayor consideración puesto que esta zona permanece valores menores en las otras mediciones, sin embargo, una posible causa de estas elevaciones de temperaturas puede ser una condición climática de ese día en particular puesto que no se realizaron las mediciones simultaneas en esta temporada. Estas características climáticas, que pueden ser de generaciones naturales, posiblemente pueden ayudar o disminuir las generaciones de las islas de calor.

- Se recomienda seguir una línea de investigación sobre las islas de calor, ya sea implementando la temperatura con calidad ambiental (por ejemplo: mediciones de concentraciones de $\mathrm{CO}_{2} \mathrm{u}$ otro gas), recolectar temperaturas de las superficies (carretera, 
aceras, edificaciones, entre otras) y temperatura ambiental para realizar comparaciones, realizar el estudio de calles perpendiculares a las vías principales.

- Como medidas de mitigación a nivel estatal se propone la inclusión de normativas ambientales estatales sobre diseños de construcción y uso de materiales de construcción. Seguimiento de los planes de ordenamiento territorial de la Ciudad de Panamá y la inclusión a nivel distrital en toda la República de Panamá. Todo esto ayuda a la disminución del fenómeno de islas de calor no solo en el área descrita, sino en otras zonas urbanas y la prevención de estas en áreas en desarrollo.

\section{AGRADECIMIENTOS}

Agradecemos a Jean Guevara y Oscar Vega por su asistencia en el uso del programa $A r c G I S$. De igual forma al Dr. Cecilio Hernández del Centro Experimental de Ingeniería de la UTP, por facilitar los termo higrómetros, equipos utilizados para la medición de temperatura y a la Facultad de Ingeniería Civil por el apoyo en la calibración de los mismos.

\section{REFERENCIAS}

[1] P. A. Mirzaei, "Recent challenges in modeling of urban heat island," Sustain. Cities Soc., vol. 19, pp. 200-206, Dec. 2015.

[2] F. Ding, H. Pang, and W. Guo, "Impact of the urban heat island on residents' energy consumption: a case study of Qingdao," IOP Conf. Ser. Earth Environ. Sci., vol. 121, no. 3, p. 032026, Feb. 2018.

[3] C. O’Malley, P. A. E. Piroozfarb, E. R. P. Farr, and J. Gates, "An Investigation into Minimizing Urban Heat Island (UHI) Effects: A UK Perspective," Energy Procedia, vol. 62, pp. 72-80, Jan. 2014.

[4] United States Environmental Protection Agency, "Heat Island Impacts." [Online]. Available: https://www.epa.gov/heat-islands/heat-islandimpacts\#main-content. [Accessed: 08-Sep-2019].
[5] M. Palme, A. Lobato, and C. Carrasco, "Quantitative Analysis of Factors Contributing to Urban Heat Island Effect in Cities of Latin-American Pacific Coast," Procedia Eng., vol. 169, pp. 199-206, Jan. 2016.

[6] W. Triyuly, S. Triyadi, and S. Wonorahardjo, "A Review of Thermal Environmental Quality in Residential Areas in Tropical Cities," IOP Conf. Ser. Earth Environ. Sci., vol. 152, p. 012034, May 2018.

[7] A. Soltani and E. Sharifi, "Daily variation of urban heat island effect and its correlations to urban greenery: A case study of Adelaide," Front. Archit. Res., vol. 6, no. 4, pp. 529-538, Dec. 2017.

[8] H. Martinez, A. Quintero, and C. De la Cruz, "Resultados Finales Ampliados: Distrito de Panamá," Contraloría General de la República de Panamá. Instituto Nacional de Estadística y Censo, 2012. [Online]. Available: https://www.contraloria.gob.pa/inec/Publicaciones/subcate goria.aspx?ID_CATEGORIA=13\&ID_SUBCATEGORIA =59\&ID_IDIOMA=1. [Accessed: 08-Sep-2019].

[9] B. Alcaldía de la Ciudad de Panamá, "Estudio de Mitigación de Cambio Climático - Iniciativa de Ciudades Emergentes y Sostenibles," Panamá, 2016.

[10] MIVIOT, "Plan de Desarrollo Urbano de las Areas Metropolitanas del Pacífico y del Atlántico/ Volumen II Parte 2," Panamá, 1997.

[11] M. de V. y O. T. (MIVI), "Documento Gráfico de Zonificación de la Ciudad de Panamá."

[12] M. Abbas, B. Jason, and J.-B. Tristan, "The urban heat island effect, its causes, and mitigation, with reference to the thermal properties of asphalt concrete," J. Environ. Manage., vol. 197, pp. 522-538, 2017.

[13] C. M. Kauffman and J. M. Moran, Our Changing Climate: Introduction to climate science. Boston: American Meterorological Society, 2014.

[14] E. Krause, "Analizar el calentamiento urbano mediante kriging," Learn ArcGIS, 2019. [Online]. Available: https://learn.arcgis.com/es/projects/analyze-urban-heatusing-kriging/. 